\title{
Hirsutism will be not Considered as Sign of Progressive Disease after Autologous Transplant in POEMS Syndrome
}

\author{
Sorà $\mathrm{F}^{* 1}$, Innocenti $\mathrm{I}^{1}$, Chiusolo $\mathrm{P}^{1}$, Autore $\mathrm{F}^{1}$, Rota $\mathrm{CA}^{2}$, Luigetti $\mathrm{M}^{3}$, Sica $\mathrm{S}^{1}$, and Laurenti $\mathrm{L}^{1}$ \\ ${ }^{1}$ Fondazione Policlinico universitario A.Gemelli IRCSS istituto di Ematologia, Rome, Italy \\ ${ }^{2}$ Fondazione Policlinico universitario A.Gemelli IRCSS istituto di Endocrinologia, Rome, Italy \\ ${ }^{3}$ Fondazione Policlinico Universitario Agostino, Gemelli, UOC Neurologia, Rome, Italy
}

*Corresponding author: Sorà F, MD, Fondazione Policlinico universitario A.Gemelli IRCSS istituto di Ematologia, Rome, Italy, Fax: +39- 6-3017319, Tel: +39-6-30154278, E-mail: federica.sora@unicatt.it

Citation: Sorà F, Innocenti I, Chiusolo P, Autore F, Rota C, et al. (2017) Hirsutism will be not Considered as Sign of Progressive Disease after Autologous Transplant in POEMS Syndrome. J Gynecol Res 4(2): 203. doi: $10.15744 / 2454-3284.4 .203$

Received Date: April 09, 2018 Accepted Date: September 04, 2018 Published Date: September 06, 2018

\section{Abstract}

POEMS (polyneuropathy, organomegaly, endocrinopathy, M-protein and skin changes) syndrome is a rare multisystem disease characterized by plasma cell dyscrasia and overproduction of vascular endothelial growth factor (VEGF). Skin changes are usually multiple cutaneous angiomas, leukonychia, necrotizing vasculitis, cutaneous thickening of sclerodermiform type and hypertrichosis. VEGF is assumed to be useful in monitoring disease activity, because VEGF levels usually decrease after treatment. We described the case of a young woman with POEMS syndrome characterized by hirsutism and hypertrichosis who underwent autologous bone marrow transplantation. Evolution was hallmarked by an improvment of radiological signs and biological markers specially VEGF, while hirsutism didn't regress.

Keywords: POEMS; Autologous transplant; Hyrsutism; VEGF

\section{Introduction}

POEMS syndrome is a rare (prevalence 0.3 per 100 000) multisystemic disorder frequently mistaken for other diseases as "Chronic Inflammatory Demyelinating Neuropathy (CIDP)". The diagnosis of POEMS was made considering the concomitance of sensorimotor demyelinating polyneuropathy splenomegaly, hypothyroidism, monoclonal gammopathy, skin changes and an increase of VEGF blood level [1-3].

Ninety percent of patients had a recognized cutaneous manifestation. Hyperpigmentation and hemangioma were most common (47\%), followed by hypertrichosis (38\%); these manifestations seems to be not evaluable to the response of therapy [3-5].

Hirsutism is a frequent manifestation (26-74\%) [1] as also the skin changes that has been reported in $46-93 \%$ of the patients. The most important parameters to evaluate the patient at the diagnosis and after the therapy are: M-protein, fluorodeoxyglucose (FDG) avidity on a PET scan and VEGF plasma level [6-8].

In POEMS syndrome improvement in peripheral neuropathy, extravascular fluid overload (ascites/effusions/edema), or pulmonary function tests, are predictive of response. The most common progression events have been Forse laboratory followed by rising VEGF [9].

In May 2014 a thirty-eight years old woman was admitted in our hematology department with the diagnosis of POEMS. Her clinical history began in july 2013 when she was admitted in neurological ward with progressive weakness, tingling and numbness in lower limbs for six months.

After the electrophysiological examination suggestive for axonal demyelinating polyneuropathy the diagnosis of CIDP was made and patient received steroids therapy obtaining a little improvement of the disease and then in november 2013 for progressive strength loss and paresthesia involved the legs, causing inabilityv to walk, she received a new cycle of steroid associated to immunoglobulin therapy $(2 \mathrm{~g} / \mathrm{Kg}$ during 5 days $)$. On that time physical examination showed bilateral pedal edema, skin thickeness and an evident hypertricosis.

Cognitive functions were normal. Examination of the fundi revealed bilateral papilledema, but otherwise cranial nerve examination was unremarkable; test showed a mild trombocytosis $\left(663 \times 10^{9} /\right.$ microl $)$ with a normal iron load and hemoglobin levels. Bcr-abl 
and Jak 2 rearrangement were done and did not show any alteration. The total serum protein count and protein electrophoresis was normal (gamma 12.5\%) without monoclonal M spike. Immunoelectrophoresis using the immunofixation method revealed a monoclonal IgA lambda band. Thyroid function tests revealed mild hypothyroidism. The prolactin level was very high (56.3ng/ml normal range 3.5-26.5). Immunological and mycrobyological tests were normal a part of the positivity of $\mathrm{HbsAg}$ and anti $\mathrm{HbcAb}$ without HBV Dna reduplications. Abdominal ultrasonography showed hepatomegaly $(16 \mathrm{~cm})$ and moderate ascites. The bone marrow biopsy from right iliac crest showed increase of plasma cells (35-40\%) with light hypoplasia of normal haematopoiesis. Total bone X ray and cerebral-spine MRI did not show bone lesion. The VEGF level was high $4164 \mathrm{pg} / \mathrm{ml}$. The diagnosis of POEMS syndrome was done.

She was included in our planned protocol and she received after lamivudine prophilaxis two cycle of intermediate dose of cyclophosphamide (4800mg) monthly in June and July 2014, and autologous peripheral stem cell collection.

In August the neurological visit described a inability of the patient to move and to maintain erect position autonomally and paralysis of lower limbs and of the distal and semi-distal muscles of the upper limbs and of the neck. The areflexia was generalized sign.

In August 2014 she received autologous peripheral stem cell infusion after a conditioning chemotherapy with melphalan $200 \mathrm{mg} /$ $\mathrm{m}^{2}$. The engraftment was obtained at day 20 for neutrophils ( $\left.>500 / \mathrm{mmc}\right)$ land at day 32 for platelets count $(20 \mathrm{x} 109 / \mathrm{mmc})$.

Then patient started a long reabilitative therapy. Bone marrow biopsy was any VEGF assay returned in normal range (758pg/ $\mathrm{ml}$ ) Five months after transplantation despite a gradually neurological improvement the patient showed a dramatical worse of hypertricosis suspect for relapsing disease (Figure 1 and 2).

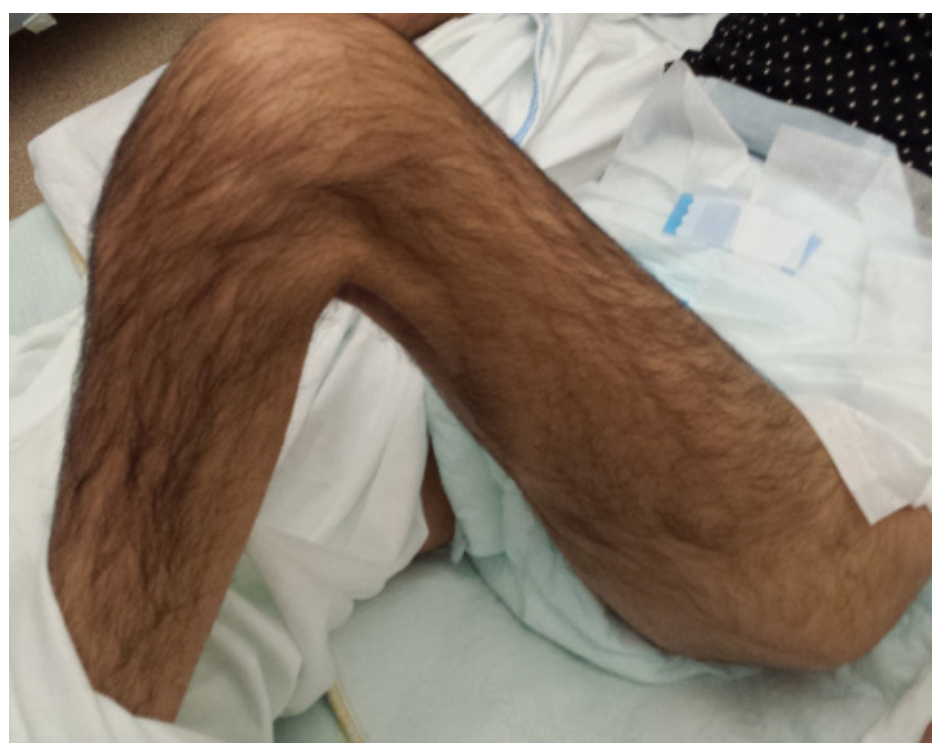

Figure 1: Patient legs after transplantation

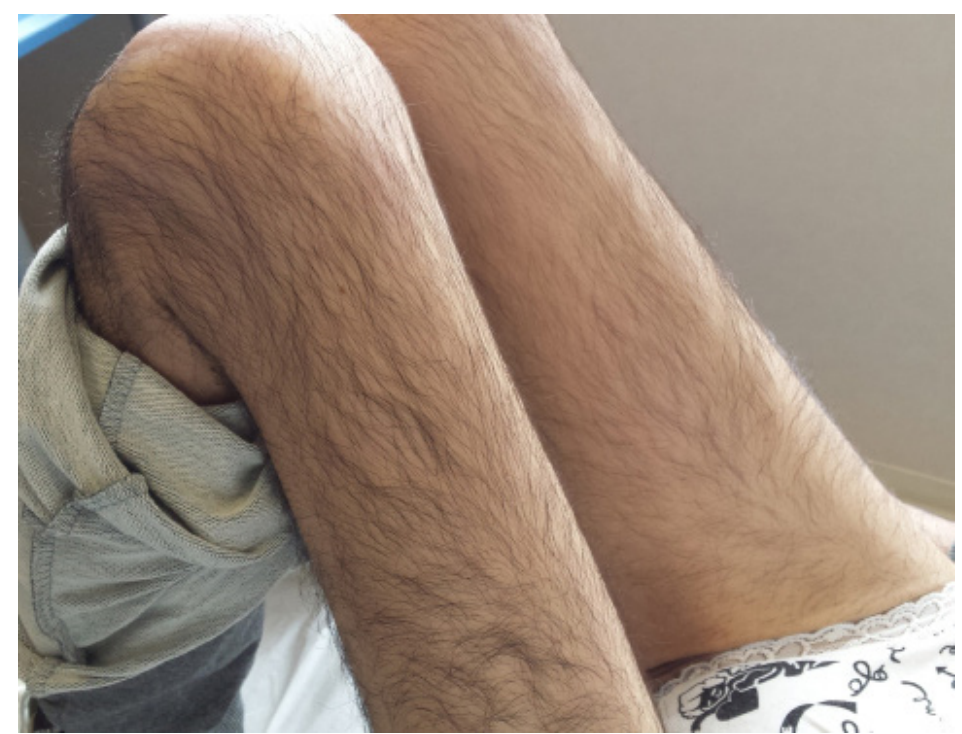

Figure 2: Patient legs after transplantation 
The haematological value and bone marrow biopsy was absolutely normal persisting only a little monoclonal gammopathy IgA lambda. Endocrinologist consultant made diagnosis of hyrsutism not related to an increased androgen levels because of the normal level of all the sex hormone (Serum hCG, FSH, LH, estradiol, prolactin, testosteron and DHEAS).

Only after eight months post transplantation the excessive body hair decreased and patient is now in complete remission 24 months from transplantation with her normal body hair (Table 1).

\begin{tabular}{|c|c|c|}
\hline & Diagnosis & After 24 months \\
\hline Monoclonal Component & IgA lambda & none \\
\hline IgA level & 1164 & 114 \\
\hline VEGF level & 4164 & 768 \\
\hline Bone marrow plasma cells \% & 20 & 2 \\
\hline Platelets count ${ }^{\star} 109 / 1$ & 663 & 239 \\
\hline
\end{tabular}

Table 1: Diagnosis after 24 months

\section{Discussion}

Here we report a case of progressive hyrsutisms appeared after 5 months from transplant who did not correlate with progressive disease. Hypertrichosis and hyrsutisms seems to be correlate with particular light chain variable region (IGLV) genes in patients with POEMS syndrome as IGLV 1-40 that had more severe neuropathy, hypertrichosis, and papilledema [10].

Patients with POEMS syndrome present with a complex conglomerate of symptoms and signs, and early diagnosis and a prompt multidisciplinary approach increase the likelihood of reduced long-term irreversible morbidity [12]. The best choice of therapy, is alkylators, either in the form of low dose conventional therapy or high dose with stem cell transplantation. Lenalidomide shows promise with manageable toxicity. Thalidomide and bortezomib also have activity, even if their risk of exacerbating the peripheral neuropathy will be conidered. The benefit of anti-VEGF antibodies is conflicting [2].

During the follow-up, the reduction or normalization of VEGF, serum M-spike, PET scan and bone marrow or peripheral blood values, will be periodically tested for its correlations with disease activity.

On the opposite, neurological problems, and skin changes need of more time to show an improvements and sometimes probably due to the therapies could show a transitory worsened. For this reason the neurological and dermatological problems do not be consider as parameter to test the response to therapy during the first follow-up; at the same time their impairment could not consider as a sign of progression.

\section{Acknowledgement}

The research are supported by "Centro di Ricerca sulle Cellule staminali emopoietiche e le terapie cellulari” Università Cattolica S.Cuore Roma

\section{Reference}

1. Rajabally YA, Seri S, Cavanna AE (2016) Neuropsychiatric manifestations in inflammatory neuropathies: A systematic review. Muscle Nerve 54: 1-8.

2. Dispenzieri A (2015) POEMS syndrome: update on diagnosis, risk-stratification, and management. Am J Hematol 10: 951-62.

3. Marinho FS, Pirmez R, Nogueira R, Cuzzi T, Sodré CT, et al. (2015) M.Cutaneous Manifestations in POEMS Syndrome: Case Report and Review. Case Rep Dermatol 7: 61-9.

4. Arana C, Pérez de León JA, Gómez-Moreno G, Pérez-Cano R, Martín Hernández T (2015) POEMS syndrome (polyneuropathy, organomegaly, endocrinopathy, monoclonal gammopathy and skin changes) treated with autologous hematopoietic stem cell transplantation: a case report and literature review. Am J Case Rep 16: $124-9$.

5. Miest RY, Comfere NI, Dispenzieri A, Lohse CM, el-Azhary RA (2013) Cutaneous manifestations in patients with POEMS syndrome. Int J Dermatol 52: 1349-56. 6. Araki N, Misawa S, Shibuya K, Ota S, Oide T, et al. (2016) POEMS syndrome and calciphylaxis: an unrecognized cause of abnormal small vessel calcification. Orphanet J Rare Dis 11: 35.

7. S Misawa, Y Sato, K Katayama, H Hanaoka, S Sawai, et al. (2015) Vascular endothelial growth factor as a predictive marker for POEMS syndrome treatment response: retrospective cohort study. BMJ Open 5.

8. Yokouchi H, Baba T, Misawa S, Sawai S, Kitahashi M, et al. (2015) Correlation between peripapillary retinal thickness and serum level of vascular endothelial growth factor in patients with POEMS syndrome. Br J Ophthalmol 100: 897-901.

9. D’Souza A, Hayman SR, Buadi F, Mauermann M, Lacy MQ, et al. (2011) The utility of plasma vascular endothelial growth factor levels in the diagnosis and follow-up of patients with POEMS syndrome. Blood 17: 4663-5.

10. Li J, Huang Z, Duan MH, Zhang W, Chen M, et al. (2012) Characterization of immunoglobulin $\lambda$ light chain variable region (IGLV) gene and its relationship with clinical features in patients with POEMS syndrome. Ann Hematol 91: 1251-5.

11. Hasegawa M, Orito H, Yamamoto K, Matsushita T, Hamaguchi Y, et al. (2012) Skin sclerosis as a manifestation of POEMS syndrome. J Dermatol 11: 922-6.

12. Dispenzieri A, Buadi FK (2013) A review of POEMS syndrome. Oncology 12: 1242-50. 


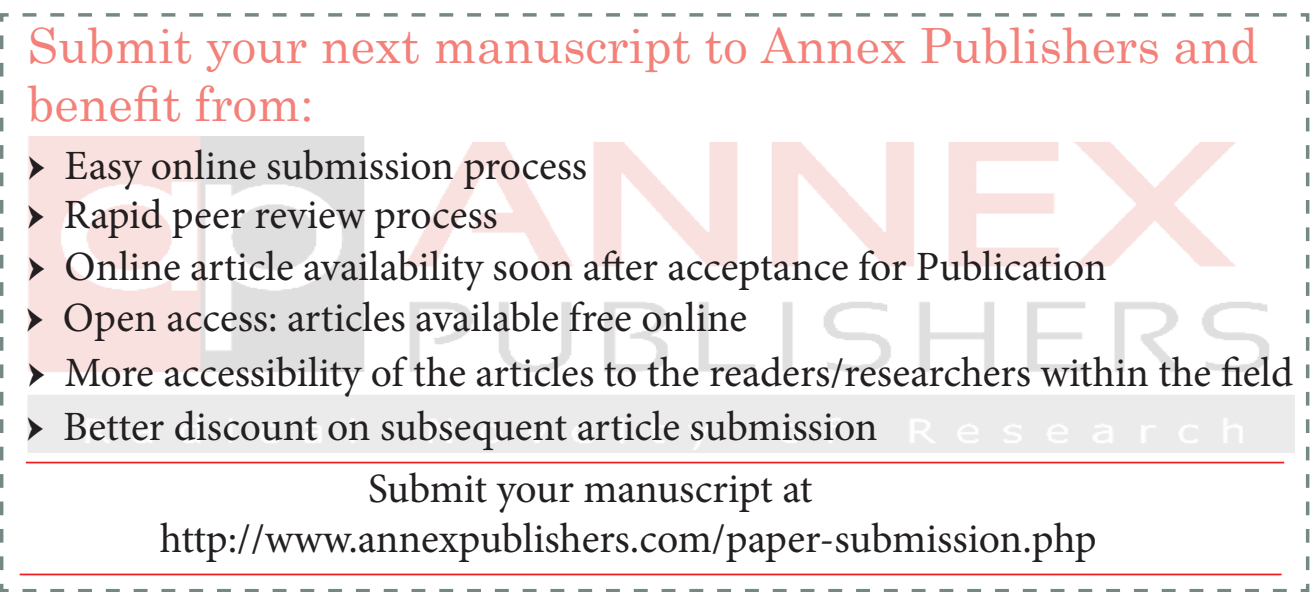

